\title{
Ultrasound-Guided Tissue Core Biopsies in Supraclavicular Lymph Nodes in Patients with Suspected Thoracic Malignancies
}

\author{
Jos A. Stigt ${ }^{a} \quad$ James E. Boers ${ }^{b}$ Martijn F. Boomsma ${ }^{c}$ \\ Departments of a Pulmonology, ${ }^{\mathrm{b}}$ Pathology and ${ }^{\mathrm{C}}$ Radiology, Isala Hospital, Zwolle, The Netherlands
}

\section{Key Words}

Biopsy · Interventional pulmonology · Histology · Cytology · Ultrasound-guided biopsy

\begin{abstract}
Background: Supraclavicular (SC) lymph node metastases are important in the analysis of thoracic malignancies for staging as well as for diagnosing purposes. Ultrasound (US) guidance visualises lesions very precisely, enabling tissue biopsies in real-time mode. Objectives: To report on the diagnostic qualities of SC tissue core biopsies (TCB). Methods: A retrospective database analysis was performed in TCB performed under US guidance in SC nodes in patients suspected of having a thoracic malignancy. Clinical characteristics and results of diagnostic evaluations were analysed. Results: Between October 2008 and October 2014, 67 sessions for TCB in 65 patients were performed. The diagnostic accuracy of TCB for all diagnoses is $90 \%$, with a sensitivity of $89 \%$. For malignant diagnoses, the sensitivity of US-guided TCB is 93\%. In 20 patients, molecular analysis for EGFR and KRAS was performed, with a diagnostic success rate of $95 \%$. One patient suffered a moderate haemorrhage after TCB. Conclusion: TCB of SC nodes in the analysis of suspected thoracic malignancy is safe and has a high diagnostic accuracy in determining tumour subtype as well as molecular analysis.
\end{abstract}

(c) 2015 S. Karger AG, Basel

\section{Introduction}

The supraclavicular (SC) areas are common sites of metastatic disease. In lung cancer, SC nodes are frequently involved and define stage IIIB disease. These nodes are easily reached and preclude more invasive investigations such as bronchoscopy or endoscopic ultrasonography in many patients [1]. Because they are often impalpable, SC metastases are primarily detected with imaging modalities such as computed tomography (CT) and positron emission tomography (PET). Ultrasound (US) is also very appropriate in demonstrating SC nodal disease but is particularly useful in guiding biopsies [2]. Under realtime guidance and with high resolution, cytologic fineneedle aspirations (FNA) or histologic tissue core biopsies (TCB) can be obtained with high precision [3]. For lung cancer staging, FNA of tumour tissue is accurate. Even for advanced elaboration of samples like histological subtyping with immunohistochemistry, FNA often provides enough material [4]. For subtyping of certain other malignancies, like malignant lymphoma, cytology is inaccurate.

Limited information is available on molecular diagnostics in FNA from SC nodes [5]. How tissue requirements for molecular analysis develop in the near future is unclear. On the one hand, there is a demand for larger amounts of material in order to enable sequential pro- 
cesses on different tumour slides. On the other hand, modern molecular tests require decreasing amounts of tissue to provide information on much more genes. It is clear from these considerations that histologic samples are not always required during the analysis of a suspected malignancy. Despite this and due to their superficial location, however, SC node metastases are ideal targets for providing large tissue samples. US guidance enables safe histologic sampling in even relatively small nodes. In this study, the diagnostic quality, regarding diagnosing as well as staging the disease, of US-guided TCB is described retrospectively.

\section{Patients and Methods}

\section{Patient Selection}

According to the interventional pulmonology database of the Isala Hospital in Zwolle, the Netherlands, a total of 278 FNA and TCB were performed. For this study, all patients who were scheduled for SC TCB are described. Screening for SC lymphadenopathy is not routinely performed in patients with enlarged mediastinal nodes, and so these patients are not included in the database. The indications for TCB were diagnosing and staging of presumed thoracic malignancies detected with PET-CT or CT alone.

The choice of TCB instead of FNA was determined by the size of the nodes, their relation with large blood vessels and the need for histology. For staging of lung cancer, FNA was considered adequate. For a diagnosis, especially when malignant lymphoma was considered, TCB was preferred. Obviously, the decision to perform TCB was influenced in time by the increasing confidence in the skills of the investigator.

The findings at physical examination, the numbers of biopsies per patient, the results of the biopsies as well as their size on CT scans were recorded. Nodal biopsies for verification of disease stage were distinguished from nodal biopsies performed only for a diagnosis. Complications of the TCBs were scored in the database and are reported as well. For this retrospective analysis, there was no ethical approval required.

\section{Tumour Measurements}

Nodal measurements during US-guided biopsies were not performed on a routine base. SC nodes that were biopsied were measured retrospectively on CT. The largest diameter in the transversal plane was used.

\section{Sampling Procedures}

A local physical examination was performed prior to US to determine if lesions were palpable or not. All biopsies were performed under local anaesthesia of the skin with lidocaine $2 \%$. Percutaneous US-guided TCB was performed with a Hitachi probe (EUP-C532) with a EZU-PA532 Hitachi Biopsy attachment. TCB was obtained under direct vision.

For histologic biopsies, $14 \mathrm{G} \times 13 \mathrm{~cm}$ Bard tissue core biopsy needles were used with a Bard magnum biopsy instrument. The disadvantage of this device is that the needle protrudes 15 or 22 $\mathrm{mm}$ (depending on the settings) into the lesion after firing the bi- opsy instrument. This was the main reason to switch to an alternative biopsy needle. Therefore, $14 \mathrm{G} \times 10 \mathrm{~cm}$ Vigeo shuttle biopsy needles were used since June 2013. This semiautomatic type of needle is inserted into the lesion and retracts upon firing. Until June 2013, the Bard TCB was used in 45 biopsy sessions in 43 different patients (2 patients with repeated biopsies), and the Vigeo shuttle biopsy needle was used thereafter in 22 patients.

\section{Pathologic and Molecular Assessment}

The biopsies were transported in formalin $4 \%$ to the Pathology Department and fixed overnight. Paraffin blocks were prepared; slides were cut and stained with hematoxylin and eosin (HE) and additional immunohistochemical staining. When non-squamous cell carcinoma of the lung was diagnosed, the TCB samples were assessed for molecular analysis. Molecular analysis was initially restricted to EGFR and KRAS genes. ALK and ROS translocations were determined in EGFR and KRAS wild types later on.

\section{Results}

Between October 2008 and October 2014, 67 US-guided investigations with TCBs were performed in 65 patients ( 2 patients underwent repeated TCBs; one after an inconclusive first session and the other to obtain additional material for molecular analysis). The patient and node characteristics are described in table 1 . The diagnoses are described in table 2.

In 47/67 (70\%) TCB sessions, a primary pulmonary malignancy was found. Non-pulmonary malignancies were found in 9/67 (13\%) patients, and in 1 patient, the biopsies showed an undifferentiated malignancy not reducible to any primary organ, and histologic subtyping was impossible. In 2/67 (3\%) investigations, benign disease was found, and in 8/67 (12\%), the samples were non-diagnostic. In $7 / 8$ patients with non-diagnostic TCBs, the result was false negative. In $1 / 8$, the biopsies could be considered as true negative as there was no SC node visible after radiologic review. In 5 patients, a diagnosis was obtained with FNA preceding the biopsies ( 3 patients with carcinomas and 2 with sarcoidosis). In 1 patient, a malignant lymphoma was found in a surgical biopsy, and in 1 patient, sarcoidosis was diagnosed on clinical grounds. Regarding these results, the diagnostic accuracy of TCB for all diagnoses is $90 \%$, with a sensitivity of $89 \%$ and a specificity of $100 \%$. For malignant diagnoses, the sensitivity of US-guided TCB is $93 \%$.

Molecular analysis of TCB was performed in 20/24 patients with a diagnosis of non-squamous cell carcinoma. EGFR and KRAS analysis was not successful in 1/20 patients (diagnostic success rate 95\%). ALK analysis was 
Table 1. Patient and node characteristics

\begin{tabular}{|c|c|}
\hline \multicolumn{2}{|l|}{ Number of biopsies } \\
\hline Range & $1-4$ \\
\hline Median & 2 \\
\hline \multicolumn{2}{|l|}{ Sex* } \\
\hline Male & 38 \\
\hline Female & 27 \\
\hline \multicolumn{2}{|l|}{ Age } \\
\hline Range & $24-82$ years \\
\hline Median & 68 years \\
\hline \multicolumn{2}{|l|}{ Location } \\
\hline Right side & 39 \\
\hline Left side & 28 \\
\hline \multicolumn{2}{|l|}{ Physical examination* } \\
\hline Positive & 29 \\
\hline Negative & 34 \\
\hline Unknown & 2 \\
\hline \multicolumn{2}{|l|}{ Imaging } \\
\hline PET positive & 44 \\
\hline $\mathrm{CT}$ & 22 \\
\hline Biopsies before imaging & 1 \\
\hline \multicolumn{2}{|l|}{ Size of nodes on CT } \\
\hline Median & $27.1 \mathrm{~mm}$ \\
\hline Range & $5.9-47.4 \mathrm{~mm}$ \\
\hline Mean & $26.6 \mathrm{~mm}$ \\
\hline \multicolumn{2}{|l|}{ Defined disease stage } \\
\hline Yes & 19 \\
\hline No & 31 \\
\hline Not applicable & 17 \\
\hline
\end{tabular}

performed in 10/16, and 1 of these analyses was unsuccessful (diagnostic success rate 90\%). One patient (blood vessel tissue in biopsy specimen) had a haemorrhage treated with local compression.

\section{Discussion}

This retrospective study demonstrates the good diagnostic features of TCB in SC lymphadenopathy. Large biopsies are easily obtained because of the superficial position and precise visualization of nodes with US. In many patients, this simple test results in a diagnosis and tumour stage as well. Due to real-time US guidance in combination with high-resolution imaging and easy distinction with vascular structures, the complication rate is extremely low. Regarding these features, it is curious that false-negative samples contained skin, fat and muscle tissue (5/8 patients).
Table 2. Diagnoses obtained with tissue core biopsies

\begin{tabular}{lr} 
Tumours of pulmonary origin & \\
Non-small cell lung cancer & 23 \\
Adenocarcinoma & 5 \\
Squamous cell carcinoma & 1 \\
Not otherwise specified & 1 \\
No further subtyping performed & 16 \\
Small cell lung cancer & 1 \\
Large-cell neuroendocrine tumour & \\
Tumours of non-pulmonary origin & 7 \\
$\quad$ Malignant lymphoma & 1 \\
Metastatic renal cell carcinoma & 1 \\
Metastatic urothelial cell carcinoma & \\
Tumour of unknown origin & 1 \\
$\quad$ Undifferentiated malignancy of unknown origin & \\
Benign diagnoses & 1 \\
Sarcoidosis & 1 \\
Fibromatosis & 8 \\
Non-diagnostic biopsies & 67 \\
\hline Total & \\
\hline
\end{tabular}

Non-diagnostic biopsies: necrosis, skin, blood vessel, inadequate material.

The 93\% sensitivity to diagnose malignancy with TCB in this series equals the sensitivity in a previous report on FNA sampling in PET-detected SC nodes (93\%) [1]. The contribution of malignant lymphoma in the study groups significantly influences the sensitivity rates. Malignant lymphoma is generally a diagnosis hard to make on cytology, resulting in relatively more false-negative FNA samples. The above-mentioned FNA series contained only 1 patient, whereas this TCB population contained 7 patients with malignant lymphoma. This is also illustrated in a previous report showing a major effect of 5 false-negative malignant lymphomas on the sensitivity rate in FNA-detected malignancies, although the sensitivity was still 97\% [6].

Molecular analysis was adequately performed on TCB in this study, with only $1 / 24$ failure for EGFR and KRAS and only $1 / 10$ failure for ALK. There are no comparative studies looking at differences in diagnostic performance of molecular tests in SC TCB versus FNA. Molecular analyses on FNA are feasible, although reports on specific SC material are limited $[5,7]$. Obviously, the results are depending on tumour cell content and contamination with other cells per aspirate and number of needle passes. Regarding the size of biopsies, one might expect more tumour cells in TCB, but it is not clear if this results in superior molecular analysis. This study shows that nodes 
even smaller than $10 \mathrm{~mm}$ are accessible for TCB. Less than half of the nodes were found on palpation, conforming to previous reports on the analysis of SC nodes detected with (PET-)CT [1]. The low sensitivity of palpation compared to US or CT was demonstrated before in a study showing $8 \%$ metastases with US-guided FNA in potentially operable patients with lung cancer [8].

In our experience, patients generally prefer sampling of SC nodes over endoscopic tests. For diagnosing and staging of lung cancer, US-guided FNA is often appropriate. Although it was never demonstrated that TCB is su- perior to FNA in diagnosing lung cancer, we prefer TCB in our clinical practice unless nodes are closely related to large blood vessels or when only staging is required. Theoretical advantages of TCB are the availability of tissue structure and larger volumes of tumour material that could enable further analysis in the future when perhaps new biomarkers become relevant for new therapies.

It is concluded that US-guided TCB of SC nodes in the analysis of suspected thoracic malignancy has a high diagnostic accuracy in determining tumour subtype as well as molecular analysis and is very safe to perform.

\section{References}

1 Stigt JA, Oostdijk AH, Boers JE, van den Berg JW, Groen HJ: Percutaneous ultrasoundguided biopsies in the evaluation of thoracic tumours after PET-CT: a prospective diagnostic study. Respiration 2012;83:45-52.

$>2$ Fultz PJ, Harrow AR, Elvey SP, et al: Sonographically guided biopsy of supraclavicular lymph nodes: a simple alternative to lung biopsy and other more invasive procedures. AJR Am J Roentgenol 2003;180:1403-1409.

$>3$ Stigt JA, Groen HJ: Percutaneous ultrasonography as imaging modality and sampling guide for pulmonologists. Respiration 2014; 87:441-451.
Gupta RK, Naran S, Lallu S, Fauck R: The diagnostic value of fine needle aspiration cytology (FNAC) in the assessment of palpable supraclavicular lymph nodes: a study of 218 cases. Cytopathology 2003;14:201-207.

5 Ellison G, Zhu G, Moulis A, Dearden S, Speake G, McCormack R: EGFR mutation testing in lung cancer: a review of available methods and their use for analysis of tumour tissue and cytology samples. J Clin Pathol 2013;66:79-89.

6 Ellison E, LaPuerta P, Martin SE: Supraclavicular masses: results of a series of 309 cases biopsied by fine needle aspiration. Head Neck 1999;21:239-246.
Lozano MD, Zulueta JJ, Echeveste JI, et al: Assessment of epidermal growth factor receptor and K-ras mutation status in cytological stained smears of non-small cell lung cancer patients: correlation with clinical outcomes. Oncologist 2011;16:877-885.

$\checkmark 8$ Sihoe AD, Lee TW, Ahuja AT, Yim AP: Should cervical ultrasonography be a routine staging investigation for lung cancer patients with impalpable cervical lymph nodes? Eur J Cardiothorac Surg 2004;25:486-491. 